\title{
Provoked vulvodynia: diagnosis of perplexing pain condition
}

\author{
๑ JACOB BORNSTEIN ${ }^{1}$, ๑ PETER PETROS², ๑ SANDY BORNSTEIN ${ }^{3}$ \\ 1Department of Obstetrics and Gynecology, Galilee Medical Center and Azrieli Faculty of Medicine, Bar-Ilan University, Nahariya, Israel \\ 2School of Mechanical and Chemical Engineering, University of Western Australia, Perth, Western Australia \\ 3Sackler Faculty of Medicine, Tel-Aviv University, Tel-Aviv, Israel
}

\begin{abstract}
Provoked vulvodynia typically presents with pain during intercourse. The etiology of this condition has not been elucidated, and the diagnostic procedures are unfamiliar to many health care providers. In a series of papers in Pelviperineology, we aimed to present the up-todate paradigms of this condition. The present paper is the first to present a contemporary approach to the diagnosis of vulvodynia based on medical history, physical examination, and the use of specific instruments and tests, based on a recent consensus terminology of vulvodynia. Management options will be discussed in another issue.
\end{abstract}

Keywords: Provoked vulvodynia; vestibulitis; vestibulodynia; pelvic floor dysfunction; chronic pelvic pain

\section{INTRODUCTION}

Provoked vulvodynia (PV) is a challenging condition for pelvic floor experts. ${ }^{1}$ Vulvodynia is currently defined as a vulvar pain of at least three months, without a clear identifiable cause, which may have potential associated factors.,3 It was previously termed vestibulitis, vestibulodynia, or burning vulva syndrome. The etiology, pathophysiology, and treatment of this condition have not been elucidated. PV may be one of the various chronic pelvic pain conditions or results from local neuroproliferation. ${ }^{4}$ The most common presentation of vulvodynia is PV, with severe entry dyspareunia, sometimes preventing the possibility to have intercourse. Over the years, women have been told that the pain during an attempted intercourse is all "in their head", which leads to despair and depression.

Given the enigma of vulvodynia, myths about its causes and treatments have emerged. However, in 2015, an evidence-based consensus terminology was introduced by scientific organizations dealing with vulvar disease - "consensus terminology", 2,3 providing a clear definition and understanding of vulvar pain. The consensus terminology included a list of eight associated factors, each of which may be the cause of the condition. This paved the way for a change in the paradigm of PV treatment, which should be tailored according to the associated factor detected at diagnosis. The approach to diagnosis has also been amended by the introduction of the consensus terminology.

In previous issues of Pelviperineology, we presented the consensus terminology ${ }^{3}$, and the associated vestibular neuroproliferation. ${ }^{4}$ The present paper is the first to expound on the contemporary approach to the diagnosis of vulvodynia. Management options will be discussed in another issue.

\section{How frequent is provoked vulvodynia?}

$\mathrm{PV}$ is a condition that women frequently conceal because they are ashamed to admit to it; thus, vulvar pain is considered to

Address for Correspondence: Jacob Bornstein, Department of Obstetrics and Gynaecology, Galilee Medical Center and Azrieli Faculty of Medicine, Bar-Ilan University, Nahariya, Israel

E-mail: mdjacob@gmail.com ORCID ID: orcid.org/0000-0003-1932-5270

Received: 24 September 2020 Accepted: 23 October 2020

${ }^{\circ}$ Copyright 2020 by the International Society for Pelviperineology / Pelviperineology published by Galenos Publishing House. 
be rare. However, the current prevalence of PV is $14 \%$ to $34 \%$ in young women and $6.5 \%$ to $45 \%$ in old women. ${ }^{5,6}$ In the United States, $30 \%$ of women reported pain during vaginal penetration. In the National Health and Social Life Survey, $21 \%, 13 \%, 13 \%$, and $8 \%$ of women aged 18-19, 30-39, and 40-49, and 50-59 years, respectively, complained of "physical pain during intercourse during the past 12 months". Another study found that $17 \%$ of postmenopausal women suffer from PV. Women of Hispanic origin are more likely to develop vulvar pain symptoms as compared with Caucasian women. ${ }^{7-12}$

\section{How to evaluate patients with vulvar pain?}

Women with vulvar pain usually go through years of endless visits to health care providers without achieving any significant improvement. This may lead to depression and suspicion in the capability of anybody to cure them. This condition preoccupies their thoughts and negatively affects their whole life. Therefore, the approach should be very empathic. Interestingly, when the diagnosis of PV is made, many patients feel relieved as they finally have a title to their condition. We suggest using a structured questionnaire (Table 1) to obtain the relevant history.

\section{Essentials of medical history:}

1. Pain descriptors, such as time since onset, temporal pattern, duration, location, quality, cause, intensity, and primary or secondary pain (Table 2). ${ }^{13}$

2. Concomitant pelvic musculoskeletal conditions, such as history of surgery or injury affecting the lumbo-pelvic-hip region and sacrum.

3. Bowel and bladder function history; disturbances may be a clue to pelvic floor dysfunction. The integral theory questionnaire should be used for this evaluation. ${ }^{14}$

4. Sexuality: desire, orgasm, and frequency of sex are a good measure of the severity of the condition.

5. Coexistence of "comorbidities" - other medical or mental health conditions and treatments.

6. Previous treatments of vulvar pain and the outcome.

7. Childhood trauma including abuse and neglect, and any adult negative sexual experience.

8. In a research setting, use of the "Initiative on Methods, Measurement, and Pain Assessment in Clinical Trials (IMMPACT)" series of questionnaires (Table 3). ${ }^{15}$

\section{Examining the vestibule}

\section{Cotton-swab test}

The cotton-swab test, also known as the Q-tip test, is an easyto-use test for diagnosing PV. At this time, a biopsy is not required to make a diagnosis of vulvodynia. Hence, we rely on clinical diagnosis. The cotton-swab test involves pressing on foci throughout the vestibule with a cotton-tipped applicator. ${ }^{16}$ Some recommend that the cotton tip be moist to avoid a painful dry sensation. We pressed vestibular foci in a non-consecutive order in the following seven vestibular foci, according to the clock's position: 1, 2 (Figure 1), 4, 6, 8 (Figure 2), 10, 11, and 12 o'clock (Figure 3). Foci 1 and 11 are located on both sides of the urethra. In addition to the vestibule, the surrounding tissues in the labia majora, perineum, and clitoris should also be touched with the cotton swab to exclude a generalized condition. The resultant pain is scored with a visual analog scale score by observing

Table 1. Structured interview of a patient with suspected provoked vulvodynia. "Have you ever had..." (based on reference 1)

Physical, sexual, and emotional abuse or anxiety?

Low back or hip pain?

Urinary urgency, frequency, hesitancy, or incomplete bladder emptying?

Chronic constipation or rectal fissures?

Oral contraceptive pill use before or during onset of symptoms?

Ovarian suppression by GnRH agonists or Depo-Provera?

Decreased libido or decreased vaginal lubrication prior to the onset of dyspareunia?

Peri-menopausal or menopausal symptoms such as hot flashes and night sweats?

Contact allergies or skin sensitive to chemicals?

Recurrent (culture positive) yeast infections?

Persistent vaginal discharge?

Severe burning or an allergic reaction to a topical medication on the vulva or vagina?

Burning after intercourse?

Pain since first attempt at intercourse without any pain-free sex?

Pain with first tampon use?

Increased sensitivity of the umbilicus?

Postcoital spotting or bleeding?

Vulvar itching, day or night?

Vulvar ulcerations, tears, fissures?

Painful periods?

Chronic pelvic pain?

Pain beginning after childbirth?

Changes in coloration or architecture of the labia or vulva?

Decreased clitoral sensation?

Pain mainly at clitoris?

Has there been any pain-free intercourse?

Oral aphthae or a diagnosis of lichen planus?

GnRH: Gonadotropin-releasing hormone 
the patient's reactions or asking the patient to rate the pain intensity on a numerical rating scale of 0 to 10 . However, this test is subjective. It depends not only on the patient's tenderness but also on the amount of pressure exerted by the examiner. ${ }^{17}$ Notably, the openings of Skene's glands, which are located lateral to the urethra and of the Bartholin's glands, may be found sensitive even in women without PV.

Location of the sensitivity, posterior versus anterior vestibular portion: Early investigators of the condition believed that, in most cases, only the posterior portion of the vestibule is affected..$^{16}$ This is not true. With time, most women with sensitivity localized only to the posterior vestibule later develop anterior allodynia. Because of this myth, during many surgical procedures for PV, only the posterior part is excised. The sensitivity later extends to the anterior part, rendering the procedure a failure. This inadequate technique is the cause of the bad reputation of the surgical treatment of PV - vestibulectomy, in the past.

An additional myth is that if the sensitivity is extensive throughout the entire vestibule, it resulted from neuroproliferation and if it is confined to the posterior vestibule, the etiology is musculoskeletal..$^{18}$ In our experience, the localization is not associated with etiology.

\section{Vulva algesiometer}

A vulvar algesiometer measures the amount of pressure causing pain applied to the vestibule in the cotton-topped test, allowing standardization of it (Figure 4). ${ }^{19}$ The pressed foci may be the same as recommended with the Q tip test.

\section{Characteristics of the pain}

The possible presentations of pain are few. Allodynia is the perception of pain resulting from a stimulus that is normally not painful, such as a cotton-swab. Hyperalgesia is the perception of extreme pain to a stimulus that is usually causing slight pain, and hyperpathia is pain provoked by very light touch. ${ }^{20}$

\section{Determining severity}

The patient usually requests to get an opinion on the severity of her PV. However, the severity is determined by the subjective level of pain at vaginal intercourse by using the Marinoff's criteria: ${ }^{20}$

Level 1: Dyspareunia causes discomfort but does not prevent sexual intercourse.

Level 2: Dyspareunia sometimes prevents sexual intercourse.

\begin{tabular}{|c|c|c|}
\hline $\begin{array}{l}\text { Pain } \\
\text { characteristic }\end{array}$ & $\begin{array}{l}\text { Core outcome } \\
\text { measure }\end{array}$ & $\begin{array}{l}\text { Secondary outcome } \\
\text { measure }\end{array}$ \\
\hline Pain intensity & $\begin{array}{l}\text { 11-point NRS during } \\
\text { sexual activities }\end{array}$ & - \\
\hline $\begin{array}{l}\text { Pain quality } \\
\text { and effect }\end{array}$ & $\begin{array}{l}\text { Short-form McGill } \\
\text { Pain Questionnaire }\end{array}$ & - \\
\hline $\begin{array}{l}\text { Pain } \\
\text { temporality }\end{array}$ & - & $\begin{array}{l}\text { Specific activities that } \\
\text { might provoke the pain } \\
\text { of PV }\end{array}$ \\
\hline
\end{tabular}

IMMPACT: Initiative on Methods, Measurement, and Pain Assessment in Clinical Trials, NRS: Numeric rating scale, PV: Provoked vulvodynia

\begin{tabular}{|l|l|l|}
\hline \multicolumn{2}{|l|}{ Table 2. Definitions of the descriptors of vulvodynia (based on reference 13) } \\
\hline Descriptor & Definition \\
\hline \multirow{4}{*}{ Location } & Localized & Involvement of a portion of the vulva, such as the vestibule (vestibulodynia), clitoris (clitorodynia), etc. \\
\cline { 2 - 3 } Provocation & Generalized & Involvement of the whole vulva. \\
\hline \multirow{4}{*}{ Onset } & Provoked & $\begin{array}{l}\text { The discomfort is provoked by physical contact. Such contact may be sexual, non-sexual or both, i.e., } \\
\text { vaginal penetration, clothing pressure, tampon insertion, cotton-tipped applicator pressure, fingertip } \\
\text { pressure, etc. }\end{array}$ \\
\cline { 2 - 3 } & Spontaneous & The symptoms occur without any provoking physical contact. \\
\hline \multirow{4}{*}{$\begin{array}{l}\text { Temporal } \\
\text { pattern }\end{array}$} & Primary & $\begin{array}{l}\text { Onset of the symptoms occurs with first provoking physical contact (i.e., tampon placement, } \\
\text { intercourse, vaginal penetration) or the symptoms are present since first recollection. }\end{array}$ \\
\cline { 2 - 3 } & Secondary & $\begin{array}{l}\text { Onset of the symptoms did not occur with first provoking physical contact or the symptoms have not } \\
\text { always been present. }\end{array}$ \\
\cline { 2 - 3 } & Persistent & $\begin{array}{l}\text { The condition persists over a period of at least 3 months (symptoms can be constant or intermittent). } \\
\text { Synonymous to chronic condition. }\end{array}$ \\
\cline { 2 - 3 } & Intermittent & The symptoms are always present. \\
\cline { 2 - 3 } & Immediate & The symptoms are not always present. \\
\cline { 2 - 3 } & Delayed & The symptoms occur during the provoking physical contact. \\
\hline
\end{tabular}


Level 3: Dyspareunia completely prevents sexual intercourse. However, when intercourse is not practiced, the tampon test

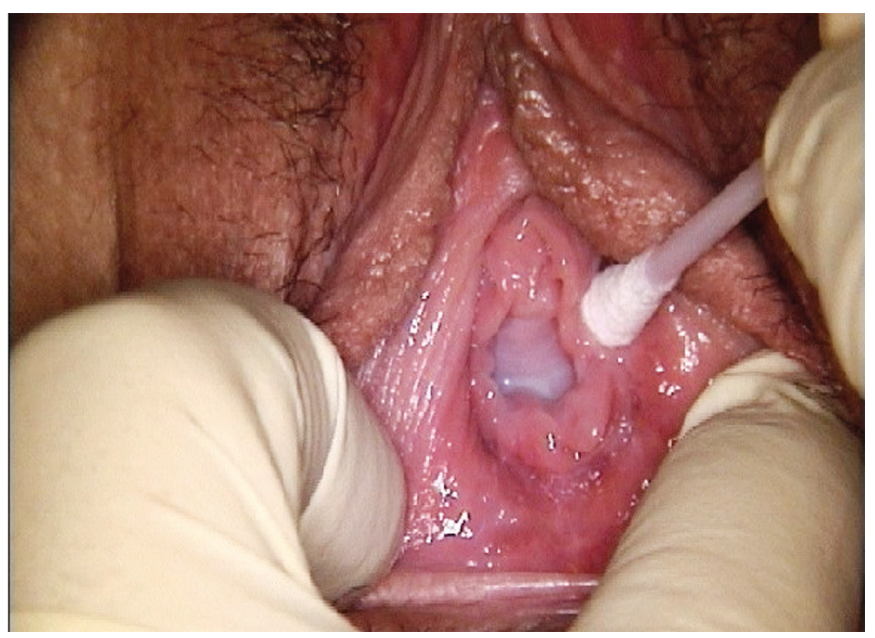

Figure 1. Q-tip swab test, pressing on the 2 o'clock focus in the vestibule



Figure 2. Q-tip swab test, pressing on the 8 o'clock focus in the vestibule



Figure 3. Q-tip swab test, pressing on the 12 o'clock focus in the vestibule may be performed by inserting a tampon to determine the severity of $\mathrm{PV}{ }^{21}$

Although this is a subjective determination, it affects the choice of treatment. In level 1 cases, treatment should not involve surgery. However, the level of pain increases with time, and the PV evaluated as level 1 may become level 2 or 3 in severity. A spontaneous regression of level 3 is rare. ${ }^{22}$ Response to treatment is also measured by reduction of dyspareunia level.

\section{Association with vulvovaginal infection}

Many women with PV have been diagnosed with vulvovaginal candidiasis (VVC). In the past, this co-occurrence originated from the theory that VVC causes PV. ${ }^{23}$ Interestingly, in mice, repeated vulvar applications of a yeast allergen (Zymosan) cause a few neuroproliferation and local sensitivity, ${ }^{24}$ serving as an animal model for PV. However, prolonged treatment with oral fluconazole has not been shown to cure PV..$^{25}$ The frequent association between VVC and PV is a myth. We suspect that the common diagnosis of VVC in women with PV results when health care providers examine a woman with entry dyspareunia and detect a slight vaginal discharge, since they are unfamiliar with the diagnosis of PV. The woman then believes that she suffers from a breakout of VVC every time she experiences pain during intercourse. This leads to repeated cycles of topical therapy with imidazole preparations against VVC, with no real improvement in pain level. Sometimes a woman with PV obviously suffers from VVC. However, VVC is incidental, and PV persists after treatment with imidazole; the woman continues to suffer from dyspareunia. This chain of events (i.e., dyspareunia, "diagnosis" of VVC, treatment with topical azoles, persistent dyspareunia) has even



Figure 4. Illustration of a vulvar algesiometer. The cotton-swab applicator is connected to a spring manometer, measuring the pressure applied. The 8 o'clock area is pressed 
led to a hypothesis that repeated administration of VVC therapy rather than VVC causes PV via an allergic reaction. ${ }^{26}$ Currently, this theory also seems a myth, resulting from VVC overdiagnosis and repeated imidazole treatment rather than PV diagnosis in women with vulvar discomfort and pain in an attempt to provide a possible explanation for the pain by a health care provider who is not acquainted with PV condition.

\section{Hormonal contraception and PV}

Hormonal oral contraceptive pills (OCP) have been claimed to be associated with an increase in PV incidence and severity. ${ }^{27}$ OCPs infrequently lead to vaginal dryness and dyspareunia. Only a minority of the millions of women who use OCPs complain of dyspareunia. Furthermore, many women with level 3 PV do not use OCPs.

\section{Excluding other causes of vulvar pain}

The first part of the consensus terminology contains a list of recognized causes of vulvar pain, including Candida and bacteriologic vaginal infections, dermatoses, deformities, birth lacerations, and past trauma. ${ }^{2}$ These should be excluded before a diagnosis of vulvodynia can be made.

\section{Colposcopic examination}

Experts of vulvar disease differ in opinions as to whether colposcopic examination of the vulva, commonly referred to as "vulvoscopy", should be a part of vulvar examination. The traditional purpose of colposcopy after acetic acid application is to evaluate the cervix in a patient with abnormal pap test, looking for intraepithelial neoplasia. On the vulva, naked eye examination may suffice in many cases. However, colposcopy is performed by many experts to magnify the vestibule and look for vulvar lesions in women with human papilloma virus lesions, suspecting vulvar neoplasia in cases of PV. The exact painful vestibular foci can be localized with the aid of a colposcope, and a fissure may be found in the posterior fourchette. We found that when women with level 3 (severe) PV attempt intercourse, forced penile insertion leads numerous times to an erosion or ulcer (Figure 5) in the fourchette. This fissure sometimes bleeds with intercourse. It may be the most disturbing painful area and the presenting symptom of PV.

In addition, colposcopic magnification can aid in excluding the presence of intraepithelial neoplasia, inflammation, infection, or any other dermatologic diseases of the vulva. Erythema is a nonspecific finding, and although it was one of the classic "Friedrich's clinical criteria"for diagnosing PV, ${ }^{16}$ it should not be used as a criterion for diagnosing PV.

\section{Speculum examination}

Performing a speculum examination of the vagina and cervix is often difficult or impossible because of entry sensitivity. It may be deferred until after the patient is more relaxed or improved with treatment. If a speculum examination was performed, any abnormal discharge should be evaluated for vaginitis. Women who are lactating, on birth control pills, or menopausal may have atrophic vaginal mucosa, which may be sensitive and cause or exacerbate vestibular sensitivity. However, the presence of any vaginal infection or atrophy does not exclude PV. They may coexist with PV, rather than being the cause of it.

Pelvic manual examination is essential for initial evaluation because it may help to determine which associated factor is present and needs attention. Inserting one finger to the vagina is less painful than two fingers, and it can suffice to evaluate the vagina, cervix, uterus, bladder, and pelvic musculoskeletal structures.

Digital pelvic examination is very important and should include the following:

- A gentle palpation of the urethra and bladder trigone. Tenderness of the bladder may be suggestive of either a urinary tract infection, a painful bladder syndrome (formerly interstitial cystitis), or endometriosis infiltration.

- Palpation of the deep pelvic musculoskeletal structures for the presence of tender points and hypertonicity. If these



Figure 5. Erosion in the 6 o'clock area of the vestibule, which is frequently caused by forceful intercourse in women with PV. It may bleed and hurt for a few days

PV: Provoked vulvodynia 
are detected, a hypertonic floor muscle dysfunction may be suspected, and a referral to pelvic floor assessment and possible rehabilitative physical therapy are recommended.

- The area around the ischial spine should be touched to detect the pudendal nerve. Tenderness of the pudendal nerve may suggest that the reason for the pain is pudendal neuralgia or pudendal nerve entrapment.

- The uterus and adnexa are palpated and moved to rule out pelvic inflammatory disease.

- If endometriosis is suspected, a recto-vaginal examination may reveal nodularity and sensitivity of endometriosis.

Repaired lacerations or episiotomies should be examined for sensitivity that may result from traumatic neuromas that can also be a source of pain in women who have had prior vaginal surgery. However, the notion that birth lacerations and episiotomy scars are a cause of dyspareunia is a myth. Postpartum dyspareunia results from the new onset of PV, while the scar tissue itself is not sensitive at all because the nerve nociceptors have been torn and destroyed in these foci.

\section{Assessment of the pelvis}

Some women with PV may have an increase in pelvic floor muscle tone. ${ }^{28}$ Interestingly, when the patient is asked to contract the introitus during a pelvic examination, the examiner hardly feels any contraction of the introital muscles, e.g., the bulbocavernosus muscle. However, the inner parts of the levator ani are usually contracted. The exact mechanism by which the instability of the pelvic floor is associated with PV is unclear. Nevertheless, detecting a musculoskeletal factor associated with PV requires physical therapy rehabilitation of the pelvic floor muscles.

Furthermore, our group described another pelvic floor abnormality in women with PV; the inability of lax uterosacral ligaments (USLS) to support the adjoining T11/L2 and S2-4 nerve plexuses may cause PV. ${ }^{29,30}$ We documented the involvement of these nerve plexuses in $\mathrm{PV},{ }^{31,32}$ and showed in a randomized, control, crossover study that support of the USLS leads to temporary relief of the PV. ${ }^{30}$ Hence, our group suggests that temporal support of the posterior vaginal fornix with a wide cotton swab or the posterior speculum blade, and repeating the Q-tip swab test ${ }^{30}$ (Figure 6) should be a part of the routine examination of every patient with suspected PV. Diminution or disappearance of the vestibular sensitivity with this maneuver attests to the role of lax USLS in the pathogenesis of PV.

\section{Pathology of PV}

A biopsy is not required for the diagnosis of vestibulodynia; however, as detailed in a previous publication in Pelviperineology, ${ }^{4}$ the four pathological features typical for vestibulodynia in biopsy are as follows:

- Stromal hyperinnervation: the stromal nerve cell surface area was found to be 10 times that of controls $(p=0.01){ }^{33}$

- Intraepithelial innervation: PGP 9.5 immunoreactive stain

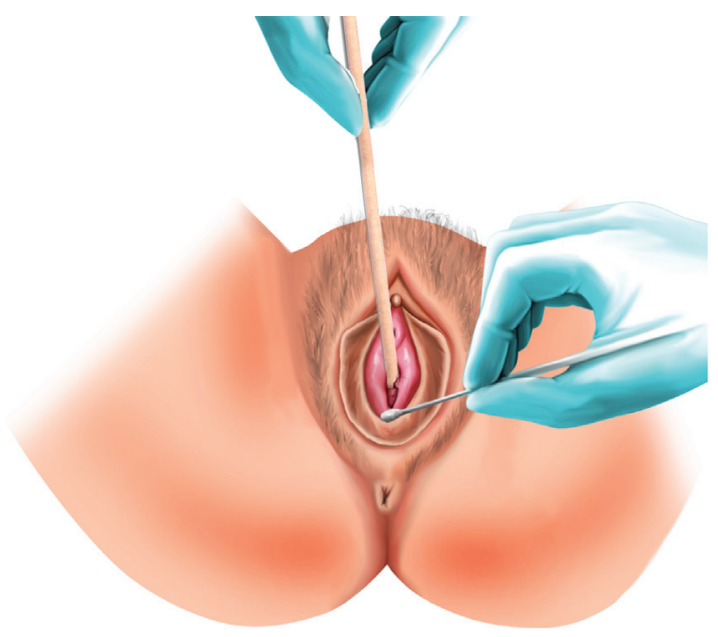

Figure 6. Q-tip swab test is performed, while the wide swab stick is in the posterior fornix

shows intraepithelial nerve fibers only in women with PV. The fibers penetrate the basal membrane and continue vertically for more than half the distance to the epithelial surface. ${ }^{34}$

- Stromal inflammation localized around the minor vestibular minor glands. ${ }^{4}$

- Increased number of stromal mast cells. Our group showed that $90 \%$ of patients with PV have more than eight mast cells per high power field..$^{33}$ The detection of mast cells may be performed using Giemsa staining or immunohistochemistry detecting CD117 (c-kit) antigen.

\section{Serum testing}

A reciprocal relationship between estrogen and vaginal innervation was observed in rodents..$^{28}$ In women with one of the conditions associated with vulvodynia - genitourinary syndrome of menopause (formerly atrophic vaginitis), a decreased serum estradiol level and a high level of follicle-stimulating hormone may be found. Elevated serum hormone-binding globulin and decreased free testosterone and estradiol levels may be detected in women with PV using OCP. ${ }^{28}$

\section{Is any additional testing required?}

In most cases, vestibular examination is sufficient, and no other test is required to diagnose PV. However, additional testing may be necessary in the following women: 
- In women with associated deep dyspareunia or deep pelvic pain, vaginal ultrasound examination and possibly magnetic resonance imaging studies should be carried out to diagnose endometriosis.

- Diagnostic laparoscopy may be necessary if a significant evidence of deep endometriosis is present.

- If nerve entrapment or compression is suspected, Tesla 3 magnetic resonance imaging using "nerve protocol" may be contributory.

- Colonoscopy and computed tomography scan with orally ingested contrast material may be used to rule out pathology of the lower gastrointestinal tract.

- Cystoscopy may be performed to diagnose painful bladder syndrome (interstitial cystitis) if PV is associated with lower abdominal pain, dysuria, etc.

- An electromyogram may be utilized to assess the tone and strength of the levator ani muscles when an evidence of hypertonic pelvic floor dysfunction is present.

\section{Determining the associated factor}

The paradigm shift that came about with the introduction of the consensus terminology and the associated factors of vulvodynia necessitates determining the management according to the factor associated with PV.

Based on the workup detailed above, we can determine the specific factors associated with vulvodynia in most cases (Table 4). In the event that a few factors seem to be associated with PV, an effort should be made to establish one cardinal factor. Treating the main associated factor often times leads to resolution of other minor factors, so the therapeutic effort should concentrate on the main associated factor and not be "multi-disciplinary." This is the case where a neuroproliferative PV is successfully treated by vestibulectomy; frequently any associated psychological factor is resolved without exclusive treatment.

Moreover, we further argue that one of the untoward befalling in the history of this condition occurs when health care providers link every dyspareunia with a psychological cause - "it is in your head" or as having a "multi-disciplinary" etiopathology, offering a fixed treatment program without concerning the unique characteristics of the specific presentation. This approach repeatedly blocks any breakthrough in the study of this perplexed condition.

\section{CONCLUSION}

Diagnosing PV should be conducted according to a structured plan. Diagnosis is based on the understanding of the associated factors of PV as described in the consensus terminology. Informing the patient of the diagnosis of PV usually relieves a lot of uncertainty from her after many years of suffering from unexplained extreme pain with each attempted vaginal intercourse. It is a required step to tailoring a successful treatment.

\section{Ethics}

Peer-review: Externally peer-reviewed.

\begin{tabular}{|c|c|c|}
\hline \multicolumn{2}{|c|}{ Associated factor ${ }^{13}$} & Determinants ${ }^{28}$ \\
\hline \multicolumn{2}{|c|}{$\begin{array}{l}\text { Comorbidities and } \\
\text { other pain syndromes }\end{array}$} & $\begin{array}{l}\text { Painful bladder syndrome, fibromyalgia, irritable bowel syndrome, and } \\
\text { temporomandibular disorder }\end{array}$ \\
\hline \multicolumn{2}{|l|}{ Genetics } & $\begin{array}{l}\text { A familial history of vulvodynia. Polymorphism c.945G }>C \text { ( } r s 222747) \text { of the gene TRPV1 } \\
\text { and a SNP in the promoter region of the gene of NGF (rs11102930) }\end{array}$ \\
\hline \multicolumn{2}{|c|}{ Hormonal factors } & $\begin{array}{l}\text { Long term oral contraceptive pills usage or genitourinary syndrome of the menopause }{ }^{27} \\
\text { Low serum Estradiol or high serum level of follicular stimulating hormone } e^{28}\end{array}$ \\
\hline \multicolumn{2}{|l|}{ Inflammation } & $\begin{array}{l}\text { Biopsy showing an increased number of mast cells, reduced systemic number of natural } \\
\text { killer cells. Repeated history of proven vulvovaginal candidiasis }{ }^{23}\end{array}$ \\
\hline \multicolumn{2}{|c|}{ Musculoskeletal } & Pelvic muscle instability, myofascial sensitivity, Uterosacral ligaments laxity ${ }^{29,30}$ \\
\hline \multirow{2}{*}{$\begin{array}{l}\text { Neurologic } \\
\text { mechanisms }\end{array}$} & $\begin{array}{l}\text { Central } \\
\text { (spinal and brain-related) }\end{array}$ & $\begin{array}{l}\text { Structural and functional magnetic resonance imaging studies demonstrating an } \\
\text { increased grey matter volume in the basal ganglia, sensorimotor cortices and the } \\
\text { hippocampus. }\end{array}$ \\
\hline & $\begin{array}{l}\text { Peripheral neuro- } \\
\text { proliferation }\end{array}$ & Biopsy revealing hyperinnervation by PGP 9.5 stain $^{4,33,34}$ \\
\hline \multicolumn{2}{|c|}{ Psychosocial factors } & Impaired mood, interpersonal, coping roles, and sexual function \\
\hline \multicolumn{2}{|c|}{ Structural defects } & Perineal descent \\
\hline
\end{tabular}




\section{DISCLOSURES}

Conflict of Interest: There are no conflicts of interest to declare.

Financial Disclosure: The authors declared that this study received no financial support.

\section{REFERENCES}

1. Bornstein J. Vulvar pain and vulvodynia. In: Bornstein J, ed. Vulvar Disease. 2019; 343-67. 1st ed. Cham, Switzerland: Springer Nature.

2. Bornstein J, Goldstein AT, Stockdale CK, et al. 2015 ISSVD, ISSWSH, and IPPS consensus terminology and classification of persistent vulvar pain and vulvodynia. J Lower Gen Tract Dis 2016; 20: 126-30.

3. Bornstein J. The consensus terminology of persistent vulvar pain and vulvodynia. Pelviperineology 2018; 37: 3-5.

4. Bornstein J, Palzur E. Provoked vulvodynia: a peripheral neurological pain syndrome. Pelviperineology 2020; 39: 68-76.

5. Bornstein J, Maman M, Abramovici H. Primary versus secondary vestibulitis - one disease, two variants. Am J Obstet Gynecol 2001; 184: 28-31.

6. van Lankveld JJDM, Granot M, Weijmar Schultz WCM, et al. Women's sexual pain disorders. J Sex Med 2010; 7: 615-31.

7. Reed BD, Harlow SD, Sen A, et al. Prevalence and demographic characteristics of vulvodynia in a population-based sample. Am J Obstet Gynecol 2012; 206: 170.e1-9.

8. Reed BD, Haefner HK, Sen A, Gorenflo DW. Vulvodynia incidence and remission rates among adult women: a 2-year follow-up study. Obstet Gynecol 2008; 112: 231-7.

9. Reed BD, Crawford S, Couper M, Cave C, Haefner HK. Pain at the vulvar vestibule: a web-based survey. J Low Genit Tract Dis 2004; 8: 48-57.

10. Harlow BL, Stewart EG. A population-based assessment of chronic unexplained vulvar pain: have we underestimated the prevalence of vulvodynia? J Am Med Womens Assoc (1972) 2003; 58: 82-8.

11. Arnold LD, Bachmann GA, Rosen R, Rhoads GG. Assessment of vulvodynia symptoms in a sample of US women: a prevalence survey with a nested case control study. Am J Obstet Gynecol 2007; 196: 128.e1-6.

12. Harlow B, Kunitz C, Nguyen R, Rydell S, Turner R, MacLehose R. Prevalence of symptoms consistent with a diagnosis of vulvodynia: population-based estimates from 2 geographic regions. Am J Obstet Gynecol 2014; 210: 40.e1-8.

13. Bornstein J, Preti M, Simon JA, et al. A multisocietal definition consensus (International Society for the Study of Vulvovaginal Disease, the International Society for the Study of Women Sexual Health, and the International Pelvic Pain Society). J Low Genit Tract Dis 2019; 23: 161-3.

14. Wagenlehner FME, Frohlich O, Bschleipfer T, Weidner W, Perletti G. The integral theory system questionnaire: an anatomically directed questionnaire to determine pelvic floor dysfunctions in women. World J Urol 2014; 32: 769-81.

15. Turk DC, Dworkin RH, Allen RR, et al. Core outcome domains for chronic pain clinical trials: IMMPACT recommendations. Pain 2003; 106: 337-45.
16. Friedrich EG Jr. Vulvar vestibulitis syndrome. J Reprod Med 1987; 32: 110-4.

17. Pukall CF, Binik YM, Khalifé S, Amsel R, Abbott FV. Vestibular tactile and pain thresholds in women with vulvar vestibulitis syndrome. Pain 2002; 96: 163-75.

18. Reed BD, Plegue MA, Harlow SD, Haefner HK, Sen A. Does degree of vulvar sensitivity predict vulvodynia characteristics and prognosis? J Pain 2017; 18: 113-23.

19. Pukall CF, Binik YM, Khalifé S. A new instrument for pain assessment in vulvar vestibulitis syndrome. J Sex Marital Ther 2004; 30: 69-78.

20. Marinoff SC, Turner ML. Vulvar vestibulitis syndrome. Dermatol Clin 1992; 10: 435-44.

21. Foster D, Kotok M, Huang L, et al. The tampon test for vulvodynia treatment outcomes research: reliability, construct validity, and responsiveness. Obstet Gynecol 2009; 113: 825-32.

22. Reed BD, Harlow SD, Plegue MA, Sen A. Remission, relapse, and persistence of vulvodynia: a longitudinal population-based study. J Womens Health (Larchmt) 2016; 25: 276-83.

23. Smith EM, Ritchie JM, Galask R, Pugh EE, Jia J, Ricks-McGillan J. Case-control study of vulvar vestibulitis risk associated with genital infections. Infect Dis Obstet Gynecol 2002; 10: 193-202.

24. Farmer MA, Taylor AM, Bailey AL, et al. Repeated vulvovaginal fungal infections cause persistent pain in a mouse model of vulvodynia. Sci Transl Med 2011; 3: 101ra91.

25. Bornstein J, Livnat G, Stolar Z, Abramovici H. Pure versus complicated vulvar vestibulitis: a randomized trial of fluconazole treatment. Gynecol Obstet Invest 2000; 50: 194-7.

26. Marinoff SC, Turner ML. Hypersensitivity to vaginal candidiasis or treatment vehicles in the pathogenesis of minor vestibular gland syndrome. J Reprod Med 1986; 31: 796-9.

27. Burrows LJ, Basha M, Goldstein AT. The effects of hormonal contraceptives on 33 female sexuality: a review. J Sex Med 2012; 9: 2213-23.

28. Bergeron S, Reed BD, Wesselmann U, Bohm-Starke N. Vulvodynia. Nat Rev Dis Primers 2020; 6: 36.

29. Petros P, Bornstein J. Re: vulvar vestibulitis may be a referred pain arising from laxity in the uterosacral ligaments: a hypothesis based on three prospective case reports. Aust N Z J Obstet Gynaecol 2004; 44: 484-5.

30. Schonfeld M, Petros P, Bornstein J. Mechanically supporting uterosacral ligaments for the relief of provoked vulvodynia: a randomised pilot trial. doi: 10.22541/au.160029751.13887485

31. Bornstein J, Zarfati D, Petros P. Causation of vulvar vestibulitis. Aust N Z J Obstet Gynaecol 2005; 45: 538-9.

32. Zarfati D, Petros P. The Bornstein test-a local anaesthetic technique for testing uterosacral nerve plexus origins of chronic pelvic pain. Pelviperineology 2017; 36: 89-91.

33. Bornstein J, Goldshmid N, Sabo E. Hyperinnervation and mast cell activation may be used as histopathologic diagnostic criteria for vulvar vestibulitis. Obstet Gynecol Invest 2004; 58: 171-8.

34. Bornstein J, Cohen Y, Zarfati D, Sela S, Ophir E. Involvement of heparanase in the pathogenesis of localized vulvodynia. Int J Gynecol Pathol 2008; 27: 136-41. 